\title{
DESTINOS DE INMIGRANTES. ESPECIALIZACIO- NES PROFESIONALES EN INMIGRACIÓN. EL CASO DE LOS MARFILENOS AGENTES DE SEGURIDAD EN LE HAVRE Y POITIERS (FRANCIA).
}

\author{
Kouadio Alexis LALLY \\ Universidad Peleforo Gon Coulibaly de Korhogo, Costa de Marfil,
}

Recibido: 3/9/2014

Aceptado: 3/11/2014

RESUMEN: La policía de siempre ha sido percibida como el único actor de la seguridad inetrior en Francia pero desde hace unos años, las cosas han cambiado drásticamente. Al lado de los policías aparecen los agentes de seguridad privada. No hay lugar en Francia donde no aparece, la seguridad privada. La observación empírica llevada a cabo en Poitiers y en Le Havre muestra que en este sector se cuenta con un colectivo que parece haber encontrado un sector de especialización profesional. Este artículo se interesa en ellos, en su experiencia de vida, a partir de una metodología cualitativa. Esta aproximación nos permitió sacar aquellas dimensiones simbólicas que acompañan siempre las vivencias de los inmigrantes al nivel micro-social. Nuestra contribución quiere hacer visible este colectivo que nos introduce en las circunstancias de la vida de agente de seguridad.

PALABRAS CLAVES : agente de seguridad/Costa de Marfil/inmigrantes/subsaharianos

ABSTRACT: The police, always, has been perceived like the only actor of the internal security in France but for some years, things have changed drastically. Next to the police appear agentes of private security. There is no place in France where the private security doesn't appear. The empirical observation carried out in Poitiers and Le Havre sample that in this sector is counted on a group that seems to have found a sector of professional specialization. This article is interested in them, in its experience of life, from a qualitative methodology. This approach allowed to remove those symbolic dimensions that always accompany the experiences of the immigrants at a microsocial level. Our contribution wants to make visible the collective that introduces us in the eventful journeys of the life of the security agent.

KEY WORDS: security agent/ Ivoru Coast/ immigrants/susaharian. 


\title{
INTRODUCCIÓN Y CUESTIONES METODOLÓGICAS
}

\begin{abstract}
¿El guardia de seguridad ?, un hombre pagado con el salario mínimo (cuando tiene los papeles) para quedarse de pie. Un trabajo que consiste en dar una impresión de seguridad. Un trabajo que parece estar reservado a los negros ... porque tienen el físico para el empleo.
\end{abstract}

Gauz (2014) Debout-Payé, Paris ; Le nouvel Attila, 192 pages .

El siglo XX ha sido un hito en la inmigración francesa ya que a sus principios, se hace

la primera llamada a la mano de obra, entonces europea, para alimentar la minas del Creusot y las industrias de acero Schneider. El fenómeno ha ido incrementándose ya sea para acompañar la reconstrucción posguerras (14-18 y 39-45) o alimentar el crecimiento de los Treinta Gloriosos, este periodo de gran crecimiento económico que conocieron la mayoría de los países desarrollados. Los flujos se hicieron contantes con la llegada de refugiados y por las reagrupaciones familiares y, sobre todo, se observó una variedad de nacionalidades. A los polacos y a los italianos, se añadieron los españoles, los portugueses, la mano de obra magrebí y negro-africana y más recientemente los turcos y asiáticos. Francia, como muchos países de la OCDE, a pesar de la crisis ${ }^{1}$ mantiene un nivel de atractivo para los africanos, abocados a una crisis endémica y a una presión demográfica que obliga a dicha inmigración. Una vez en Francia, la necesidad de supervivencia les obliga a buscar trabajo en sectores que les son asequibles y que a menudo, obedecen a enclaves profesionales.

Como señala SUZANNE (2014; 88) el origen determina muy a menudo los empleos que los inmigrantes desempeñan en los lugares de acogida $\mathrm{y}$, precisamente, la cuestión de las profesiones basadas en el origen étnico ha sido tratada por varios investigadores. En España, por ejemplo, ODDEN (2006) se interesa en los senegaleses en la venta ambulante en Salamanca.

En el Líbano, según MENHEM (2014), los inmigrantes de Sri Lanka, de Nepal, de Filipinas, de Etiopía, de Bangladesh, se especializan en los trabajos domésticos, cuando los sirios, los egipcios y los indios trabajan en la construcción, en la agricultura o en la industria.

\footnotetext{
${ }^{1}$ La crisis financiera actual muestra que las migraciones son muy sensibles a los contextos políticos y sobre todo económicos. En periodos de recesión, incluso países que parecían haber sobrellevado las dificultades económicas vuelven a las andadas. Españoles y portugueses retoman las sendas hacia otros países europeos por el paro.
} 
LILLO $(2011)^{2}$ relata el caso de los italianos, españoles y portugueses en Francia. También en esta pauta CYIMPAYE (2001; pp.220-228) ${ }^{3}$, que trabajó sobre este colectivo en este país, asegura que los inmigrantes de esta nacionalidad que vinieron a Costa de Marfil se especializaron en la actividad agrícola para luego migrar hacia empleos asalariados como el trabajo de guardia, puerta de entrada de las actividades urbanas. HILY Y RINAUDO ${ }^{4}$ (2002) hicieron lo propio en Italia con los senegaleses. En Francia, SALL $(2001)^{5}$ se centra en los senegaleses, especialistas de la venta ambulante en París. Podremos citar decenas de investigaciones pero nuestro propósito aquí es otro. Nos interesa la seguridad privada un sector en el que aparecen muchos inmigrantes subsaharianos y que todavía no ha sido investigado a fondo. De los pocos estudios que hay, podemos citar el de BONNET (2008) quién trabajó sobre los efectos perversos del reparto de la seguridad entre la policía pública y privada tomando como ámbito de observación una estación y un centro comercial. En un trabajo anterior, HUG (2000) señala que en el sector de la seguridad privada, la demanda es fundamentalmente fenotípica con negros africanos como protagonistas. Aunque nuestro trabajo se inspira en las aproximaciones de este autor, la perspectiva aquí desarrollada se distingue por su objeto de estudio que es exclusivamente el colectivo marfileño.

Nuestra investigación busca conocer el ámbito de la seguridad privada a partir del colectivo marfileño que se ha especializado en este sector. Hemos hecho una descripción y análisis de la presencia de los marfileños en el mercado de trabajo francés con un enfoque que toma en cuenta diferentes escalas de sus vivencias. Más específicamente, nos interesa saber las representaciones sociolaborales de este ámbito profesional a partir de la reconstrucción de las condiciones de vida de sus actores.

Para este cometido, creemos pertinente usar un enfoque metodológico cualitativo en la tradición antropológica. La investigación consistió en llevar a cabo una observación directa y realizar entrevistas en profundidad con actores

${ }^{2}$ LILLO, N.(2011) Italiens, espagnols et portugais en France au xx ème siècle, regards croisés », Révue européenne des migrations internationales, vol.27 n² (2011) en línea desde el 01 de octubre de 2014), consultado el 22 de octubre de 2014. URL : http//remi. revues.org/5500.

${ }^{3}$ CYIMPAYE, D. (2001) MIGRATION, EMPLOI ET ÉPARGNE EN AFRIQUE : le cas des migrants burkinabé à Abidjan (Côte-d'ivoire). Ottawa: National Library of Canada, p.370.

${ }^{4}$ HILY, M. A. y RINAUDO C. (2002) "Vintimille, ville marché : L'espace des cirulations et du commerce". En M.A.HILY y E. MA MUNG. (dirs.) Catégories et lieux des circulations migratoires. Rapport final pour la MIRE.Poitiers, Migrinter, pp. 63-98.

${ }^{5}$ SALL, L. (2001) Commerces et commerçants sénégalais à Paris: Étude de cas. Tesina de Licenciatura. Poitiers, Universidad de Poitiers. 
de la seguridad en todos los espacios laborales en los que nos hemos movido, al estilo de PAILLÉ Y MUCCHIELLI $(2012 ; 13)^{6}$. La idea era tener informaciones de primera mano para poder sintetizar el punto de vista de cada actor. Nuestra idea es que con una encuesta de este tipo se debe llegar a una explicación de la realidad, permitiendo una interpretación de las visiones del mundo de los actores del contexto del ámbito investigado.

La muestra se compone de 14 inmigrantes marfileños. Hemos asociado a 08 nativos franceses, entre los cuales 05 , aparecen en el cuerpo de nuestro trabajo. El objetivo es obtener su punto de vista sobre la realidad estudiada ya que lo viven en primera persona. En nuestra investigación también han participado empresarios del sector de la seguridad privada.

El trabajo empírico se llevó a cabo en varios lugares de vida de estos inmigrantes tales como sus casas y también sus lugares de trabajo tales como los centros comerciales (Géant en Poitiers y Printemps en Le Havre). Las noches romanas de Poitou-Charente ${ }^{7}$ son otro marco de ejercicio de la profesión de una manera distinta de cómo se lleva a cabo en un centro comercial o en las instalaciones de una empresa. El trabajo de campo se extendió a Costa de Marfil, país de origen de nuestro colectivo de estudio.

Como se verá a lo largo del trabajo, la codificación no es rigurosa porque aunque aparecen nombres, son ficticios para mantener un cierto anonimato solicitado por nuestros informantes.

\section{LO MARFILEÑOS EN LA SEGURIDAD PRIVADA EN FRANCIA.}

Aunque no se tiene datos estadísticos claros, la presencia numerosa de marfileños en la seguridad privada en Francia no es ningún secreto. Hasta tal punto que esta característica ha pasado a la tradición popular en las canciones de los marfileños cuando hablan de la dificultad de la inmigración en Francia ${ }^{8}$. El debate sobre este tema étnico se quedaría superficial si no consultamos a los concernidos. Llegará el momento de servirse de ellos como pretextos para hablar

\footnotetext{
${ }^{6}$ PAILLÉ,P. y MUCCHIELLI, A. (201) L'Analyse qualitative en Sciences humaines et Sociales. Paris: Armand Collin, p.13.

${ }^{7}$ Les Nuits Romaines, son un conjunto de espectáculos gratuitos que tienen como objetivo hacer descubrir el excepcional patrimonio cultural romano de la región francesa de Poitou-Charentes. Son espectáculos que movilizan un material logístico enorme que necesitan de la presencia de agentes de seguridad para velar primero por ello, y luego para vigilar el desarrollo de las representaciones

${ }^{8}$ Yodé y Siro, famosos cantantes marfileños, decían que "todos los marfileños cuando llegan a Francia se hacen agentes de seguridad incluso los delgaditos".
} 
de otros temas pero de momento, nos interesa saber su experiencia de vida, sus perspectivas y proyectos en este trabajo.

El trabajo de agente de seguridad tiene rasgos étnicos en la consecución del trabajo. La construcción de las redes profesionales se hace en base a mecanismos de captación basadas en la nacionalidad. Los marfileños con algunas otras nacionalidades africanas negras se benefician de la demanda explícita de las empresas contratistas que se enfocan en negros para su negocio en vigilancia activa.

Llegué en Francia en el 2004, buscaba algo de trabajo para vivir... un amigo mío me presentó a un conocido suyo propietario de una empresa de seguridad en Poitiers. No tenía los papeles pero me contrató al negro ... así es cómo empecé a hacer este trabajo...(Emery; 32, CM)

Antes de ir más lejos, cabe decir que la dignificación de todo trabajo puede ser función del lugar o del país de ejercicio de este trabajo. Aunque no tiene una base empírica, es extraño ver que los marfileños, que en su país no se interesaban por este trabajo, lo están ejerciendo cuando llegan a Francia. El trabajo de seguridad, más bien en su variante de vigilante en Costa de Marfil, era de los burkineses. CYIMBAYE (2001) cree que para ellos, es un modo de ascender socialmente después de trabajar en las plantaciones. Este fragmento etnográfico, denota de la veracidad de nuestra afirmación. Cissé, es otro agente que llegó a Francia como estudiante en el año 2004 pero que no pudo acabar la carrera.

Nunca pensé que iba a trabajar de eso, aqui en Francia. Ironía de la suerte, en mi casa trabajaba un guardia que es burkinés... Mi padre tenía dinero y eso propicio que llegara aqui para estudiar...y mira, hoy estoy aqui, en esta noche fría vigilando este material, mientras duerme la gente. Esto nunca lo haría en Costa de Marfil..(Cissé; CM)

Es un fenómeno que recuerda lo que pasa en España con la vendimia. Este año (2014) según recogen diversos medios de comunicación, 15.000 españoles se apuntaron a esta cosecha de uvas en Francia, una actividad que hubieran podido hacer en su país si no fuera por los mejores emolumentos que reciben en Francia. Aquí, la motivación es financiera, y puede asemejarse del todo al caso marfileño si no fuera porque en su caso, el rechazo a este trabajo es obvio en su país.

Según nuestras primeras observaciones, es un "trabajo de mierda" por las diferentes experiencias que viven.

Es un trabajo que no es fácil. Tienes que estar siempre de pie. Te insultan. Además no se respetan los horarios. Puedes pasar fácilmente siete u ocho horas con una sola hora de 
pausa. Cada seis horas deberíamos tener al menos cuarenta y cinco minutos de descanso. No se nos da. Y cuando te quejas al director de la tienda te responde "no te pago para que vengas a descansar. Sin embargo, es lo que prevé la Ley. (Mory: CM)

Este otro fragmento es de uno de nuestros informantes, Konan:

...tu empleador no te respeta, los clientes tampoco y además no te pagan bien. De verdad, puede afirmar que es un trabajo de mierda. Es sencillamente porque soy inmigrante que lo hago. Pase lo que pase, está mejor remunerado que el trabajo que tenía en mi pais. (Konan; $C M$ )

A parte de estas vejaciones morales, otras más físicas forman parte de la vida de los agentes. La violencia que pueden sufrir forma parte de los riesgos de su trabajo. Es por lo menos lo que asegura Mory:

" A veces por haber arrestado a alguien puede venir a esperarte donde vives o por donde pasas para hacerte daño. Por suerte de momento no he tenido este tipo de problemas pero algunos colegas han tenido más de una vez que pasar por el quirófano por apuñalamiento. No siempre es noticia pero el caso que si lo fue es el de un vigilante de origen marfileño que fue abatido en Ivry sur seine por haber denegado la entrada a una discoteca a jóvenes que no llevaban la ropa correcta». (Mory; CM)

¿Pero, si es un trabajo de mierda, porque atrae tanto este trabajo a los marfileños?

El marfileño suele cuidar su apariencia vestimentaria por lo que le va este trabajo. Ya Pascal HUG (2000) $)^{9}$ constató este hecho. En su estudio vio como entre los africanos, solían ser los marfileños y los congoleños, precisamente las nacionalidades que tienen fama de ser los que más se cuidan de su atuendo ${ }^{10}$.

\footnotetext{
${ }^{9} \mathrm{HuG}, \mathrm{P} .(2000)$ "Les agents de sécurité privée noirs : un exemple de discrimination dans le monde de la sécurité”. En les cahiers de la sécurité intérieure. Les emplois jeunes. Paris: INHESJ, pp.93-117.

${ }^{10}$ Los inmigrantes de los dos Congo (Kinshasa y Brazzaville) son conocidos por ir siempre elegantes con su concepto de la SAPE. «Sociedad de las Ambientadores y de las Personas Elegantes », grupo informal de jóvenes oriundos de Gongo quienes al final de los años 80 hacía acopio de vestidos de marca una vez en París para luego volver exhibirse a su vuelta según WARNIER (1999; 108). La SAPE existe ha pervivido en
} 
Este trabajo es también un lugar de refugio que les permite evitar el paro, como veremos más adelante, el trabajo manual (menos dignificante que recuerda a unos su pasado en el país de origen) y de mantener una cierta dignidad social, precisamente gracias a la elegancia del vestido de trabajo. Si la ropa, en general, es un modo de ponerse al nivel de los nativos KOUADIO (2012), también es importante el hecho de poder dar órdenes a los blancos, acostumbrados históricamente a dar órdenes a negros desde la colonización, y poder decir, yo puedo darle ordenes ahora desde mi posición.

"me sentía orgulloso de poder mandar a mi manera, hacer que los blancos obedezcan a mis órdenes. ;Date la vuelta, aquí no puedes pasar! Riéndose. Me siento fuerte.(Emery; $C M)$

Afloja aquí una expresión del complejo de inferioridad latente que siempre ha guiado al negro según FANON (1952) ${ }^{11}$. Como lo hemos observado tanto en África como en Francia, un blanco o una blanca produce en el negro un comportamiento de deferencia que es fácil relacionar con la historia colonial y al mito de la superioridad blanca todavía muy interiorizado por los negros.

¿Pero realmente que piensa el blanco de este trabajo? Un informante francés que preguntamos sobre la fuerte presencia subsahariana en la seguridad nos dijo:

...es un trabajo que no necesita muchos esfuerzos ni inteligencia...es decirno necesita una cualificación particular es ideal para los negros... (Narcisse; FR)

Si nos basamos en los requisitos para ser agente de seguridad, diríamos que no tiene nada cierto lo que afirma nuestro informante ya que se debe tener diferentes niveles de estudio para hacer este trabajo. Hay niveles base como CAP (agente de prevención y de seguridad), MC (seguridad de los espacios abiertos al público) hasta niveles de bachillerato $+2,+3$ o +5 . A eso se añaden cualificaciones profesionales como el certificado de agente de seguridad en incendios, o el certificado de operador en tele seguridad, etc.

La poca valoración ${ }^{12}$ que se tiene de este trabajo hace que casi se le deja a inmigrantes, algo difícil de ver en España. La creencia de que los agentes no

\footnotetext{
África y los marfileños también son muy conocidos con una variante de este concepto asociado al Coupe-décalé KOUADIO (2012).

${ }^{11}$ FANON, F. (1952) Peau noire masque blanc. París, Seuil.

${ }^{12}$ Preguntados sobre la necesidad de hacer este trabajo, los nativos lo consideran demasiado duro para que se interesen en él.
} 
tienen cualidades intelectuales, ni formación profesional es falsa y se debe a qué la economía sumergida encuentra en el sector uno de sus bastiones preferidos. De todos los marfileños investigados, sólo 5 tienen la formación adecuada para hacer este trabajo, los demás ejercen en la economía sumergida o sea al "negro". Los africanos que se acometen a este empleo son generalmente, gente necesitada de trabajo rápido por lo que aceptan todo lo que se les da. Acaban generalmente explotados, como en el caso de Junior, un "sin papeles" de Costa de Marfil que pudimos entrevistar durante las Noches Romanas en Melle, una pequeña ciudad de Francia:

$$
\begin{aligned}
& \text { “...hombre, un trabajo aquí es un trabajo, no puedo } \\
& \text { quejarme. Tengo que vigilar todo el material del circo que } \\
& \text { se representará dentro de dos dias. Estoy sólo y no viene el } \\
& \text { relevo. Con lo cual, tengo que hacer guardia de día y también } \\
& \text { de noche. Me pagará por ello y para mí mientras más horas, } \\
& \text { mejor. ...corro muchos riesgos para mi salud pero también } \\
& \text { me puede repatriar si me cogen porque estoy sin papeles...es } \\
& \text { un riesgo innecesario para el jefe pero lo acepta. Por eso, no } \\
& \text { puedo quejarme... " (Junior; CM) }
\end{aligned}
$$

Llegan pues, no por verdadero deseo profesional sino por necesidad puntual. Sin embargo, los que verdaderamente quieren este trabajo o quieren especializarse saben que es un sector de promoción en el empleo.

Los burkineses de Costa de Marfil cuando llegaban empezaban a trabajar en las plantaciones y uno de los trabajos a los que se acogían cuando se acercaban a las ciudades era ser guardia. Los marfileños en Poitiers empezaban también por la agricultura (cosecha de manzana, de melón, etc...) para luego ser agentes de seguridad. Ser agente de seguridad, aparte de tener un trabajo más estable aparece pues como una promoción. Dramane es un ejemplo. Le conocimos con motivo de la investigación para le Tesis doctoral en 2010. Aseguraba trabajar en la cosecha de manzana, pero años después, cuando volvimos a verle, trabajaba como agente de seguridad.

“...era muy bueno, el mejor de todos en la cosecha de
manzanas. Perecía haber nacido para este empleo pero
necesitaba algo que durara todo el año...tú después de
ganar 2000 euros en la campaña de manzanas, tienes que
buscar otra cosa para vivir y no siempre es fácil. Tienes que
moverte por todo el país en busca de cosechas. Es dificil...”
(Dramane; CM)

Respetando las pautas de las antiguas migraciones en Francia, (españoles e italianos, portugueses y magrebíes), vemos que el trabajo de agente de seguri- 
dad es algo transitorio para algunos subsaharianos. Es el caso de este estudiante marfileño de Poitiers, que aprovecha muy bien sus 1,90 metros para ser agente de seguridad:

“...yo hago este trabajo a tiempo parcial para tener medios para acabar mi tesis doctoral. En ningún modo, lo quiero como trabajo definitivo... es un sacrificio para luego trabajar en algo mejor..." (Pierre Marie; CM)

Este deseo de nuestro informante no siempre se cumple porque de nuestros informantes, tres son doctores con recorridos parecidos aunque con historias de vida diferentes. Benjamin es uno de ellos, después de sacar adelante el doctorado quiso trabajar en Francia únicamente:

“...poder, podía pero no quise volver a Costa de Marfil porque de siempre ha sido mi objetivo venir a Francia. Ahora que lo conseguí, creo que haber obtenido mi título me da posibilidades de trabajar aquí. De momento acepto este trabajo de agente de seguridad y ya veremos..." (Benjamin; CM)

Los 4 años que lleva nuestro doctor en este trabajo son pocos en comparación con los 7 de Armel, otro doctor agente de seguridad. Después de acabar la Tesis doctoral en Geografía, volvió a Costa de Marfil donde fue profesor en la Universidad. Él mismo nos cuenta como volvió a este trabajo:

“...yo lo hacía antes junto con los estudios...volví luego a Costa de Marfil cuando me saqué el título. Fui profesor en una de las Universidades públicas del país pero aquello no era lo que quería de verdad...Yo quiero trabajar aquí y no en Costa de Marfil, aunque fuese de momento como agente de seguridad..."(Armel; CM)

Puede parecer difícil creerlo pero para algunos, el estatus social no se valora tanto como los medios económicos. Las motivaciones personales ajenas son difíciles de juzgar sobre todo en nuestro caso, sencillamente porque no hemos podido obtener todos los contornos de tal decisión y sus implicaciones socioeconómicas.

Nuestro tercero Doctor, que hemos llamado Coulibaly, en cambio, trabajó a tiempo parcial también de agente de seguridad mientras era estudiante. Sus criterios en cuanto a este trabajo son otros. Sin saber que estábamos informado, nos contó el caso que mencionamos arriba antes de dar su punto de vista: 
“...un amigo mío de carrera trabajaba como profesor en la Universidad en Costa de Marfil pero lo dejó para venir a ser guardia en Francia. Hay que estar enamorado de Francia para cometer semejante tontería a pesar de lo que él cree mejor...lo habría entendido si fuera alguien que no tiene su nivel...yo lo hago cuando vengo de vacaciones para pagar mi billete de vuelta porque soy profesor en la Universidad de Abidjan..."(Coulibaly; CM)

Algunos ejemplos, arriba mencionados, resuenan como un fracaso de las políticas de retorno de los estudiantes, predicadas por los gobiernos africanos. Los intelectuales que no quieren volver se contentan pues de trabajos como el que nos ocupa porque no encuentran fácilmente trabajo en su ámbito de estudio. Entre los africanos, muchos dejan sus buenas posiciones para venir a Europa KOUADIO (2012). Otros en cambio lo hacen porque cuando la aventura sale bien, las remesas son una verdadera salvación ya que contribuyen al bienestar de la familia en origen. Es el caso por ejemplo de Marco, otro agente de seguridad, de origen marfileño, que trabaja en un Hipermercado en Le Havre:

"no sólo trabajo aquí... de día sí pero por la noche, tengo otras vacaciones para poder vivir aqui y hacer vivir mi familia que está en Costa de Marfil... hago todo eso para mis hijos, ...para que tengan mejor vida que yo. Tengo que pasar horas de pie aqui y seguir por la noche sin apenas descanso. Eso, en mi pais, no lo haría pero aqui si..." (Marco; CM)

En el día a día de este oficio, los agentes sufren frecuentes actos deliberados o no de racismo, un racismo siempre "a la vuelta de la esquina" cuando abordamos el tema de inmigración subsahariana en Europa.

\section{A VUELTAS CON EL RACISMO.}

En un país donde la igualdad entre personas y el respeto a la raza son una "religión" por ser el país de los derechos humanos, cualquier acto puede ser diversamente interpretado como racista o xenófobo si se protagoniza en contra de un negro subsahariano. Nuestra observación en Le Havre, es una muestra de ello. Un día, estuvimos por casualidad en un supermercado fuimos testigo de una escena que nos interesó porque entraba de lleno en nuestra investigación. Un hombre de cerca de 40 años entro en el supermercado. El guardia de seguridad, allí de pie en la entrada le pedía a la gente depositar sus mochilas en un armario que lleva una llave y recuperarla a la salida del local. Tuvo la desgracia de pedir lo mismo a un gran señor. Éste se negó y provocó un escándalo. ...jvaya!, decía, 
me lo pides porque soy negro ${ }^{13}$, ¿verdad? Era gracioso porque el agente también es negro. « es inaceptable. Me estás insultando...te subes a un tren y te controlan sin controlar a los demás, en la calle te paran para pedirte el documento de identidad. Entonces hay franceses a los que se controla y otros que no... y esta señora que entró antes que yo, con su bolso, le dijiste algo? : «... esta señora tiene el bolso más grande pero ella sí que puede entrar ¿verdad? Es porque soy negro...eres un acomplejado, un vendido ». ¿Por qué este escándalo por una simple demanda de depositar la mochila en un armario que tiene llave?

Aunque no pudimos hablar con el agente, otro, informante tiene método para evitar fricciones con los clientes, sean subsaharianos o no.

«... a nosotros, ya sabes, se nos borra la sonrisa rápido. Tema de racismo...Yo desde luego, les (a los negros) hablo con respeto y con la sonrisa. Es la única forma de romper el hielo. Generalmente cuando les tratas con respeto aún cuando tienen un bolso grande, te lo abren sin problema...claro, eso cuando no se llevan nada... y cuando pides a alguien enseñar su bolso, tienes que estar seguro a $100 \%$ porque luego te pueden denunciar por difamación y todo recae sobre ti porque el dueño de la tienda no te va a defender..." " (Benjamin; CM)

Sylvestre, un profesor de colegio de origen subsahariano, nos decía a propósito del racismo:

"No quiero ser un defensor de nadie pero, y es verdad, a veces nos quejamos y vemos el racismo por todos los lados... a base de ser víctimas de racismo, caemos en la paranoia. El racismo existe pero hay que saber diferenciar las cosas. Pero, te digo una cosa..., algunos empleadores usan a los negros vigilantes y les obligan aplicar reglas racistas a una parte de su clientela... Si creo que se sirven de los negros para purgar a otros negros y de este modo no se hablará de racismo..." (Sylvestre; CM)

En nuestra labor investigadora, sobresalió en una conversación con el Director de una agencia de seguridad que es francés, un rasgo que parece pertinente

\footnotetext{
${ }^{13}$ En nuestra observación participante, pudimos constatar que la relación entre negros es diferente de lo que pudimos ver en la ciudad de León donde estuvimos. A lo que nos queremos referir es que parece que cuando la población de origen aumenta de número, las relaciones ya no son tan cercanas y un negro o un blanco suelen recibir el mismo trato por de un subsahariano.
} 
para dar una explicación de la preferencia por la piel oscura a la hora de contratar a agentes de seguridad.
«...nosotros no somos partidarios de la discriminación y del racismo... al contrario. Aqui tengo a varios chicos de color, árabes $y$, por supuesto, a blancos. Cada uno puede ser útil en un contexto u otro. Llevar a blancos en un entorno donde hay muchos negros puede no ser fructifero... a veces $\mathrm{si}^{14}$, pero muchas veces no es aconsejable...sencillamente porque durante su intervención no se les tacharía enseguida de racistas...sólo porque quieren filtrar las entradas y salidas... "'(Charles; FR)

Eso es un testimonio de una de las dimensiones complejas de la gestión del racismo en el seno de la sociedad francesa. Con eso, nos enfrentamos pues a una de estas creencias que profesan los responsables de ciertas instituciones económicas americanas. Justifican el hecho de mandar a negros americanos a África con el argumento de que sus acciones tendrán más repercusión debido a su color de piel. La encuesta que hicimos a pie de hipermercados a un conjunto de informantes seleccionados por ser blancos tanto en le Havre como en Poitiers, nos dio respuestas que no difieren tanto de los fragmentos etnográficos arriba mencionados. A la consideración de si creen que hay muchos negros en la seguridad y a qué creen que se debe, muchos dieron respuestas del tipo:

- Creo que sí...porque antes cuando se cogía a los ladrones decían todo el tiempo que es porque los agentes son racistas...con una persona del mismo origen, no se puede decir que es racista y eso calma a todos. (Jacques; FR).

- no, pero un blanco neutraliza a un delincuente y en pronto se pretende víctima inocente porque en malo de racista quiere echarle la culpa de lo que no ha hecho...con un black, difícil hacer un escándalo ...(Serges; FR)

- no sé de dónde ha salido esta estadística pero no lo creo...y si hay muchos agentes negros para entornos negros, tampoco me lo creo

\footnotetext{
${ }^{14}$ Pudimos constatar, en cambio, que un agente blanco en un entorno donde se mueven muchos subsaharianos como suele haber en ciertos barrios puede ser bueno. El blanco por esta relación colonial con el africano es rígido y categórico cuando controla. No negocia mucho. En el supermercado de Caucriauville donde trabaja el informante negro, nos decía que, a veces, cierra los ojos cuando algunas mujeres con sus vestidos amplios típicos de países musulmanes esconden cosas para salir. La jugada es tan bien sabida que ni siquiera salta la alarma del pórtico. Nos decía que él sabe cómo lo hacen y sólo negocia con ellas para que no se reproduzca. Un blanco hubiera pedido la devolución inmediata sin miramientos.
} 
mucho pero supongo que es más difícil acusar de racismo a otro negro pero si es un blanquito, será acusado por asociaciones tales como SOS Racismo y demás...(Octave; FR)

Elegir a negros para combatir a negros o magrebíes puede tener otra explicación relativa a la disuasión que provocarían los agentes de seguridad negros sobre los delincuentes negros o de origen africano. Nuestra observación en Caucriauville nos permitió ver una realidad que podemos considerar disuasiva y que es el principio de "hermano mayor" es decir del tradicional principio de respecto al mayor que sigue rigiendo las relaciones entre inmigrantes más jóvenes y inmigrantes subsaharianos mayores, cualquiera que sea su nacionalidad. Esta relación es casi afectiva fenotípica e identitaria.

“...bueno, lo más importante es crear el respeto con los chicos. Solo unos pocos se muestran gamberros e intercambian palabras conmigo. Ahora ya el respeto aqui es otra cosa... Europa lo cambia todo...porque imitan a chicos de aqui que insultan a los mayores a los ancianos ${ }^{15}$ (Mory; CM)

A estas experiencias se añaden a otras relacionadas con el ejercicio de su profesión, el saber hacer frente a sus imperativos laborales, evitar hurtos y fundirse al paisaje del lugar sin suscitar desconfianza entre los clientes.

\section{EL SABER ESTAR EN EL MANTENIMIENTO DE LA SEGURIDAD.}

En un centro comercial, es verdad que hay que evitar robos, pero lo más importante es rentabilizar el negocio por el que se está vigilando. Por eso, el trabajo de un agente no debe poner trabas a la dinámica comercial. Una presencia discreta da seguridad a los compradores pero una visibilidad excesiva de los agentes puede ser mala para el negocio. La susceptibilidad de los clientes negros por ejemplo, puede hacer perder a esta franja de buenos compradores. Durante nuestra labor investigadora, salimos de compra con nuestro compañero de piso, un negro, de origen marfileño. Su reacción frente a las sospechas del agente de seguridad, blanco esta vez, nos parecería exagerado si no fuera porque abarcaba un tema que para algunos es real.

“...desde que hemos entrado, no ves que este señor nos sigue como si fuéramos ladrones. Lo que va es que nunca más

\footnotetext{
${ }^{15}$ Esto fundamentalmente se debe, entre otras cosas, a que las segundas y terceras generaciones no viven y tampoco siguen los valores africanos. Éstos se diluyen cuando el asentamiento es definitivo. Los jóvenes se identifican con la sociedad local, con otros valores entre los cuales por ejemplo, "un joven y una persona mayor son iguales ante un asiento libre en el autobús.
} 
vuelva aqui a comprar... O sea, los negros son los ladrones. Yo no he robado nunca y estas continuas sospechas en los lugares comerciales me están molestando. Tendrán sus razones pero no creo que el robo solo sea cosas de negros... (Kouamé; CM)

Flusty $(2001)^{16}$ hablando de un tema que sigue completamente vigente, decía lo siguiente: "por un lado los usuarios exigen la presencia protectora visible contra encuentros imprevisibles con la alteridad. Por el otro, se niegan a gastar su dinero en un lugar que se parece a un cuartel militar". Los agentes deben pues entrar en la lógica comercial de la tienda y eso exige comportamientos como aquellos que describe Konan:
“...en un lugar de trabajo como este, tienes que cambiar el chip. No sirve de nada ir de cachas y todo... el guardia de seguridad aquí, además de ser eficaz debe ser cortés. Tenemos que acompañar al emplear que busca que la gente venga a comprar en su almacén...si por una sospecha, se arresta a alguien inocente, la tienda es responsable de la humillación...por eso, a veces dejamos salir a la gente con duda razonable para no armar un follón entre compradores $y$ dueños de la tienda... " (Konan; CM)

En todo caso, un ambiente sereno es más propicio al consumo e incompatible con el recurso a la violencia. En realidad, como hemos podido observar, los agentes de seguridad ponen en marcha un plan de seguridad definido según los desafíos comerciales del cliente. La atención al cliente es fundamental en los órdenes que reciben del empleador. Hay que acatar estos órdenes y la protección contra el robo no es nada más que un medio para limitar las pérdidas. En ningún momento debe impedir que compren los clientes. Estas medidas hacen que los gerentes se preocupen incluso del atuendo de la higiene de los agentes que vienen. Koudougnon es marfileño, después de muchos años siendo agente de seguridad, montó su propia empresa que emplea a treinta empleados fijos. Este fragmento etnográfico es un claro testimonio de ello:

“...en ciertos lugares, nuestros agentes pueden permitirse ir
al trabajo en pantalones vaqueros y camisetas para cazar
con el entorno. En los centros comerciales, no se puede.
Hay que ir uniformados y eso contribuye a dar una imagen
buena a mi empresa y también a la persona que solicita mis

${ }^{16}$ FLUSTY, S. (2001) The Banality of Interdiction: Surveillance. Control and the Displacement of Diversity. International Journal of Urban and Regional Research 25, 658-664. 
servicios. Para mi cliente la apariencia física, un criterio de elección...(Koudougnon; CM)

\section{CONCLUSION}

Para comprender mejor el trabajo de los marfileños en Francia, hemos trabajado con los marfileños de Poitiers y Del Havre que ejercen de agentes de seguridad en Francia. Para ello, hacía falta una investigación que integrara el punto de vista de aquellos con los cuales hemos realizado esta investigación. Era necesaria la integración también, de una perspectiva de un investigador de origen africano, cuya perspectiva debía aportar elementos enriquecedores en el sector de la seguridad privada y del trabajo de los inmigrantes en general. Es lo que hemos intentado hacer, centrándonos en las vivencias de los agentes marfileños de esta nacionalidad. Así mismo, esta investigación permite entender el carácter oportuno de dejar este aspecto de la seguridad de los bienes en grandes superficies a empresas privadas y no a la policía, lo que seguramente habría provocado otro tipo de problemas. Esta investigación abre un abanico de posibilidades para entender mejor esta profesión pero deja seguramente algunas preocupaciones abiertas que, a buen seguro, tendrán repercusión entre investigadores sociales.

\section{BIBLIOGRAFÍA}

BAUER A., VENTRE A.-M. (2010). Les polices en France : sécurité publique et opérateurs privés. Paris : P.U.F. (Que sais-je ?)

BONNET F., (2008), "Les effets pervers du partage de la sécurité. Polices publiques et privées dans une gare et un centre commercial", Sociologie du travail, 4.

CYIMPAYE, D. (2001) MIGRATION, EMPLOI ET ÉPARGNE EN AFRIQUE :

le cas des migrants burkinabé à Abidjan (Côte-d'ivoire). Ottawa: National Library of Canada, p. 370

FANON, F. (1952) Peau noire masque blanc. París, Seuil.

FLUSTY, S. (2001) The Banality of Interdiction: Surveillance. Control and the Displacement of Diversity. International Journal of Urban and Regional Research 25, 658-664.

GAUZ (2014) Debout-Payé, Paris ; Le nouvel Attila, 192 pages

HUG P., (2000). «Les agents de sécurité privée noirs. Un exemple de discrimination dans le monde de la sécurité", Les cahiers de la sécurité intérieure, 40, pp. 93-118.

PÉROUMAL, F. (2008). "Le monde précaire et illégitime des agents de sécurité", Actes de la recherche en sciences sociales, $n^{\circ}$ 175(5), pp. 4-17 
KOUADIO ALEXIS, L. (2012) La inmigración subsahariana en España. Imaginarios, experiencias y perspectivas de futuro de los subsaharianos en León. León: Universidad de León, p.718.

LOUBET DEL BAYLE J.-L., (2010). "Les enjeux sociopolitiques de la sécurité privée”, Revue internationale de criminologie et de police technique et scientifique, 1 , 100-121.

MENHEM, S. (2014) Les nouveaux migrants au Liban : vers une ethnicisation du marché de l'emploi. Poitiers: emigrinter, pp. 86-90.

OCQUETEAU F. (2004). Polices entre Etat et marché, Paris, Presses de Science Po.

ODDEN, G. (2006) Les migrants sénégalais à Salamanque, stratégie de mobilité sociale et trajectoires migratoires. Poitiers, Universidad de Poitiers, Departemento de Geografía.

PAILLÉ,P. y MUCCHIELLI, A. (201) L'Analyse qualitative en Sciences humaines et Sociales. Paris: Armand Collin, p.13.

SALL, L. (2001) Commerces et commerçants sénégalais à Paris: Étude de cas. Tesina de Licenciatura. Poitiers, Universidad de Poitiers.

WARFMAN D., OCQUETEAU F., (2011). La sécurité privée en France, Paris, PUF. (Que-sais-je ?)

WARNIER (J.-P.), 1999, Construire la culture matérielle, Paris, PUF, 176p 